\title{
Functional characterization of hypertrehalosemic hormone receptor in relation to hemolymph trehalose and to oxidative stress in the cockroach Blattella germanica
}

\author{
Jia-Hsin Huang ${ }^{1}$, Xavier Bellés ${ }^{2}$ and How-Jing Lee ${ }^{1}{ }^{*}$ \\ 1 Department of Entomology, National Taiwan University, Taipei, Taiwan \\ ${ }^{2}$ CSIC-UPF, Institute of Evolutionary Biology, Barcelona, Spain
}

\section{Edited by:}

Joe Hull, USDA Agricultural Research

Service, USA

\section{Reviewed by:}

Dalibor Kodrik, University of

South Bohemia, Czech Republic

Gerd Gäde, University of Cape Town

South Africa

\section{*Correspondence:}

How-Jing Lee, Department of Entomology, National Taiwan

University, No. 1, Sec. 4, Roosevelt

Road, Taipei 106, Taiwan.

e-mail:m480@ntu.edu.tw
Hypertrehalosemic hormone $(\mathrm{HTH})$ is a peptide hormone that belongs to the adipokinetic hormone/red pigment concentrating hormone (AKH/RPCH) family, which exerts pleiotropic actions related to catabolic reaction and stress response. AKH peptides have been demonstrated to participate in stress response including oxidative stress in several insects. In order to study the signaling pathway of $\mathrm{HTH}$ involved in anti-oxidative stress, we have characterized a $\mathrm{HIH}$ receptor cDNA in Blattella germanica (Blage-HTHR) in structural and in functional terms using RNA interference (RNAi). Blage-HTHR is expressed in various female adult tissues (brain-CC-CA, ventral nerve cord, midgut, fat body, oviduct), but maximal expression is observed in the fat body. RNAi-mediated knockdown of Blage-HTHR expression results in a significantly lower level of hemolymph trehalose, even though HTH is exogenously administered. Paraquat elicits lethal oxidative stress in B. germanica, and co-injection of paraquat and $\mathrm{HTH}$ reduces this detrimental effect and extends the median survival time. Interestingly, the "rescue" effect of HTH on mortality caused by paraquat is diminished in specimens with depleted expression of Blage-HTH and Blage-HTHR. Finally, lipid peroxidation in the hemolymph increases $4 \mathrm{~h}$ after paraquat treatment, in comparison with control specimens or with HTH-treated specimens. However, lipid peroxidation induced by paraquat was not "rescued" by HTH in Blage-HTH and Blage-HTHR knockdown specimens. Our results demonstrate that HTH acts as a stress hormone mediating antioxidative protection in B. germanica, and that its receptor, Blage-HTHR, is essential for this action.

Keywords: adipokinetic hormone, paraquat, RNA interference, hypertrehalosemic hormone receptor, German cockroach

\section{INTRODUCTION}

The adipokinetic hormone/red pigment concentrating hormone $(\mathrm{AKH} / \mathrm{RPCH})$ family is one of the extensively characterized group of neuropeptides whose members play important roles on energy homeostasis, as well as on other physiological and behavioral processes in insects (Schooley et al., 2005). AKH peptides are 8to 10 -amino acid long, with a pyroglutamate blocked $\mathrm{N}$-terminus and an amidated C-terminus, aromatic residues at positions 4 and 8, and a glycine residue at position 9, when present (Gäde, 2009). Among them, the peptides that primarily induce trehalose efflux into hemolymph are referred to as a hypertrehalosemic hormone (HTH).

Recently, AKHs have been related to stress response, through mechanisms involving the stimulation of catabolic reactions while inhibiting synthetic reactions by increasing available energy (Kodrik, 2008). Earlier studies have related the release of AKHs as a result of insecticide poisoning through recording the increase of metabolites such as trehalose and lipids in the hemolymph of the cockroach Periplaneta americana (Granett and Leeling, 1972) and the locust Schistocerca gregaria (Samaranayaka, 1974). Direct measurement of AKH titer increase after insecticide exposure has been further reported in a number of insects, including the locust S. gregaria and the bug Pyrrhocoris apterus (Candy, 2002; Kodrik and Socha, 2005; Velki et al., 2011). In addition, AKHs participate in anti-oxidative mechanisms by increasing reduced glutathione efflux and decreasing protein carbonyl levels in response to treatment with paraquat $(\mathrm{PQ})$, a bipyridylium herbicide that is currently used to elicit oxidative stress in animals through redoxcycling reactions (Hassan, 1984); it has been used in this way, for example, in the potato beetle Leptinotarsa decemlineata (Kodrik et al., 2007) and in P. apterus (Vecera et al., 2007).

Oxidative stress is a type of stressor that causes neurodegeneration of brain cells under severe stress condition (Chaudhuri et al., 2007); it also affects a number of physiological processes, including an adaptive compensatory specific response of the organism that is activated to sustain homeostasis (Valko et al., 2007). Reactive oxygen species, such as superoxide anion radicals, hydroxyl radical, and hydrogen peroxide, represent a class of molecules that are derived from the normal metabolism of oxygen in the majority of aerobic organisms. Reactive oxygen species arise as endogenous by-products of metabolic reactions in the cells, and from exogenous exposure to environmental pollutants, ionizing radiation, 
and others. Excess of reactive oxygen species and/or deficit of scavenging antioxidants results in oxidative stress, which may impair correct regulation of lipids, proteins, and DNA (Halliwell and Gutteridge, 2007).

Most of the previous studies show that AKHs are involved in antioxidant protection mechanisms used biochemical experimental approaches such as monitoring $\mathrm{AKH}$ titer changes or exogenously applying AKH under oxidative challenges (Kodrik et al., 2007; Vecera et al., 2007). Thus, little information is available at the molecular level on the relation of $\mathrm{AKH}$ with antioxidant protection mechanisms. In this context, we aim to provide molecular evidence that the signaling pathway of $\mathrm{HTH}$ is involved in the antioxidant protection mechanisms, using the cockroach Blattella germanica as model. Previously, we had cloned the hypertrehalosemic hormone (Blage-HTH) cDNA in B. germanica (Huang and Lee, 2011). In the present study we report the cloning of the cDNA of the HTH receptor (Blage-HTHR) and the effect of PQ when both Blage-HTH and Blage-HTHR expression are silenced by RNA interference (RNAi) in B. germanica, a species that is particularly sensitive to this knockdown technique (Belles, 2010).

\section{MATERIALS AND METHODS INSECTS}

A colony of the German cockroach, B. germanica (L.), was maintained in environmental chambers at $28^{\circ} \mathrm{C}$ under $\mathrm{L}: \mathrm{D}=16: 8 \mathrm{~h}$ conditions. Dog chow and water were provided ad libitum. Nymphs and adults were reared in groups, but adults were separated by sex to keep them in virgin state. Detailed information about rearing has been described previously (Lee and Wu, 1994).

\section{RNA EXTRACTION AND REVERSE TRANSCRIPTION TO cDNA}

All RNA extractions were performed using TRIzol Reagent (Invitrogen) following the supplier's protocol. Possible genomic DNA contamination was removed by DNase I (Promega) treatment. One microgram of total RNA was reverse transcribed using High Capacity cDNA Reverse Transcription Kits (ABI).

\section{CLONING OF THE HTH RECEPTOR}

The first primer set was designed based on the known $\mathrm{AKH}$ receptor sequences of other insects, including $P$. americana (DQ217786), Apis mellifera (AY898652), Tribolium castaneum (DQ422965), and Bombyx mori (AF403542). The primer sequences were as follows: $5^{\prime}$-TCC ACG TGG AGG AGC ACC C$3^{\prime}$ forward; and, 5'-TTC TTC CAS STG GAG GRR CAC CCC-3' reverse. The amplified fragment ( $420 \mathrm{bp}$ ) was subcloned into the pGEMT-easy vector (Promega) and sequenced. To complete the sequence, the GeneRacer Kit (Invitrogen) for rapid amplification of cDNA ends (RACE) was used following the manufacturer's protocol. For 5'-RACE, the reverse primer was $5^{\prime}$-GAC TCA GCT GTA CCA GTT CTG GTA CAG GA-3', and for $3^{\prime}$-RACE, the forward primer was 5'-GTC TGA ACT GAG CTG ATA TCT GCG A-3' . All PCR products were subcloned into pGEMT-easy vector (Promega) and sequenced.

\section{PHYLOGENETIC ANALYSIS}

The amino acid sequences of all known insect $\mathrm{AKH}$ receptors were obtained from GenBank and aligned using the Clustal X
1.18 program (Thompson et al., 1997). The resulting alignment was manually edited using the GeneDoc program (Nicholas et al., 1997) and analyzed by the Neighbor-joining method for construction of a phylogenetic tree. The AKH receptor of the insect species with the GenBank accession numbers are Drosophila melanogaster (AAF52426), Anopheles gambiae (ABD60146), Aedes aegypti (CAY77164 and CAY77166), Glossina morsitans (AEH25943), B. mori (AAL95712), Manduca sexta (ACE00761), A. mellifera (AAX83121), Nasonia vitripennis (NP_001161243), T. castaneum (ABE02225), Acyrthosiphon pisum (XP_001945436), Pediculus humanus (EEB15485), P. americana (ABB20590), and B. germanica (ADL60118). Five-letter abbreviations used for these $\mathrm{AKH}$ receptors and species are indicated in Figure 1B. Phylogenetic trees based on the resulting alignment were constructed using MEGA version 5 (Tamura et al., 2011). The gonadotropinreleasing hormone receptor of Caenorhabditis elegans (CaeelGnRHR, GenBank accession number NP_506566) was used as outgroup. The robustness of phylogenetic analysis was evaluated by bootstrapping using 1000 replicates.

\section{BLAGE-HTHR EXPRESSION ANALYSIS}

We performed semiquantitative RT-PCR to investigate the distribution of Blage-HTHR in different tissues of 7-day-old adult female B. germanica and to assess the effects of RNAi. Thus, the $25 \mu \mathrm{lPCR}$ mixture for amplifying the Blage-HTHR fragment included $1 \mu \mathrm{l}$ of cDNA, 10 pmol of forward primer ( $5^{\prime}$-GAC TCA GCT GTA CCA GTT CTG GTA-3'), 10 pmol of reverse primer ( $5^{\prime}$-GGG AAA TGT CTT GTG AAC CAG GTC- $3^{\prime}$ ), and $5 \mu$ l of Taq polymerase mixture (Protech). The PCR (30 cycles) was performed as follows: denaturation at $94^{\circ} \mathrm{C}$ for $30 \mathrm{~s}$, annealing at $58^{\circ} \mathrm{C}$ for $30 \mathrm{~s}$, extension at $72^{\circ} \mathrm{C}$ for 1 min. Expression of BgActin (GenBank EU514491) was used as reference. $\mathrm{PCR}$ products were separated and visualized on a $1.5 \%$ agarose gel.

Quantitative real-time PCR measurements were carried out in triplicate in Applied Biosystems StepOnePlus real-time PCR systems with Fast SYBR Green Master Mix (Applied Biosystems). The primers used to measure Blage-HTHR mRNA were as follows: $5^{\prime}$ TCT ATC GGG ACA ACA GCA ACC AGA-3' forward, and 5'-TGC TTC AGG AAC TTC ACT CAC AGT- $3^{\prime}$ reverse. The efficiency of this primer set was first validated by constructing a standard curve through five serial dilutions. The expression levels were normalized to BgActin expression using 2(-Delta Delta $\mathrm{C}(\mathrm{T})$ ) method (Pfaffl, 2001). The PCR program began with a single cycle at $95^{\circ} \mathrm{C}$ for $3 \mathrm{~min}, 40 \mathrm{cycles}$ at $95^{\circ} \mathrm{C}$ for $15 \mathrm{~s}$, and $60^{\circ} \mathrm{C}$ for $30 \mathrm{~s}$. Afterward, PCR products were heated to $95^{\circ} \mathrm{C}$ for $15 \mathrm{~s}$, cooled to $60^{\circ} \mathrm{C}$ for $30 \mathrm{~s}$ and increasing the temperature $0.5^{\circ} \mathrm{C} / \mathrm{min}$ in order to measure the dissociation curves and to determine an unique PCR product for each gene. A template-free control was performed in each batch.

\section{RNA INTERFERENCE}

The Blage-HTHR cDNA of the German cockroach was first cloned into the pGEMT-easy vector (Promega, Madison, WI, USA). Then, a 528 bp fragment targeting the $3^{\prime}$-UTR of Blage-HTHR mRNA was amplified by PCR. The primers (which contained the T7 promoter sequence: TAATACGACTCACTATAG) used in this PCR reaction were as follows: $5^{\prime}$-TGG ATA GAC CAG CAG TCT GCT 
A

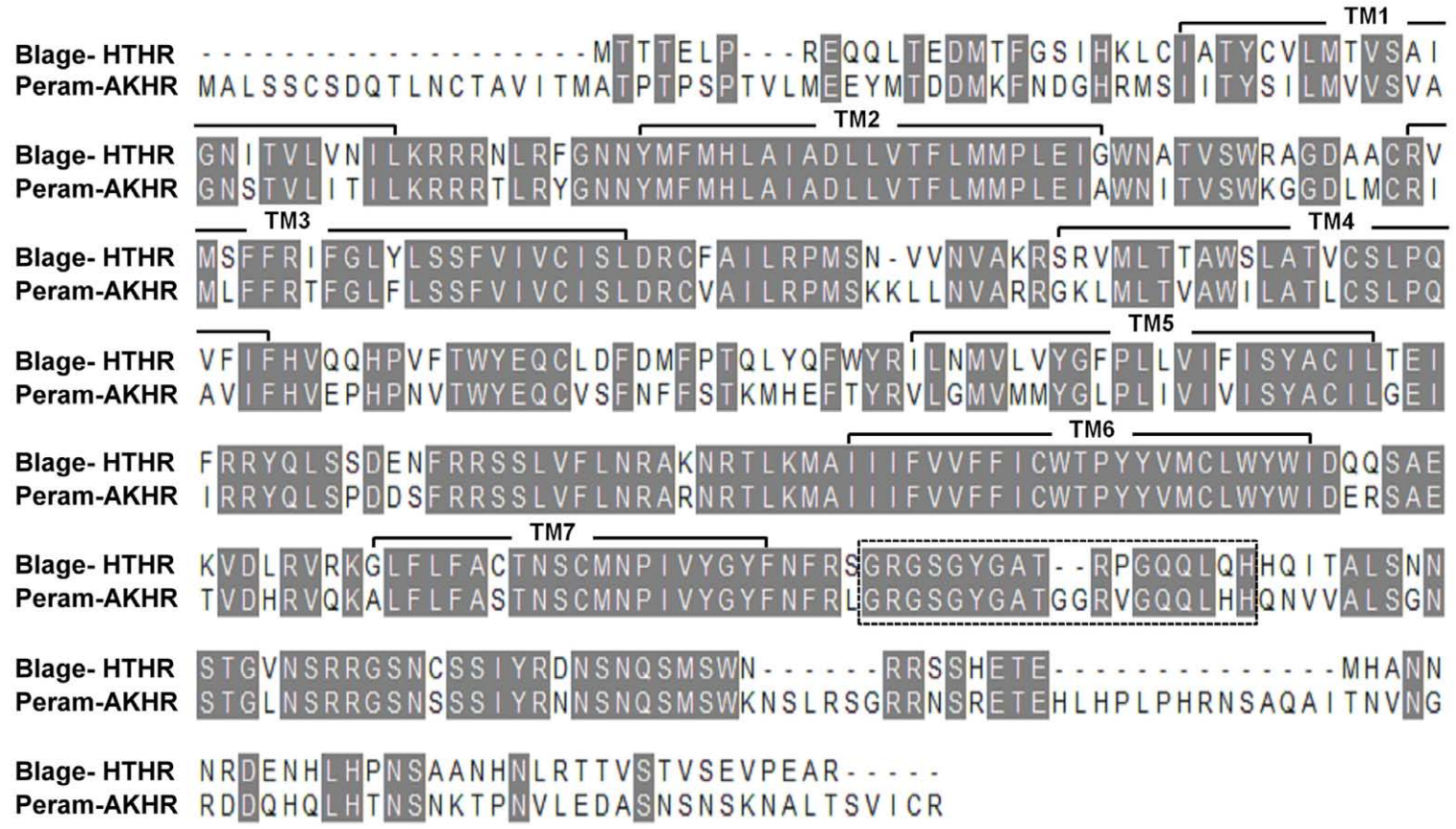

B

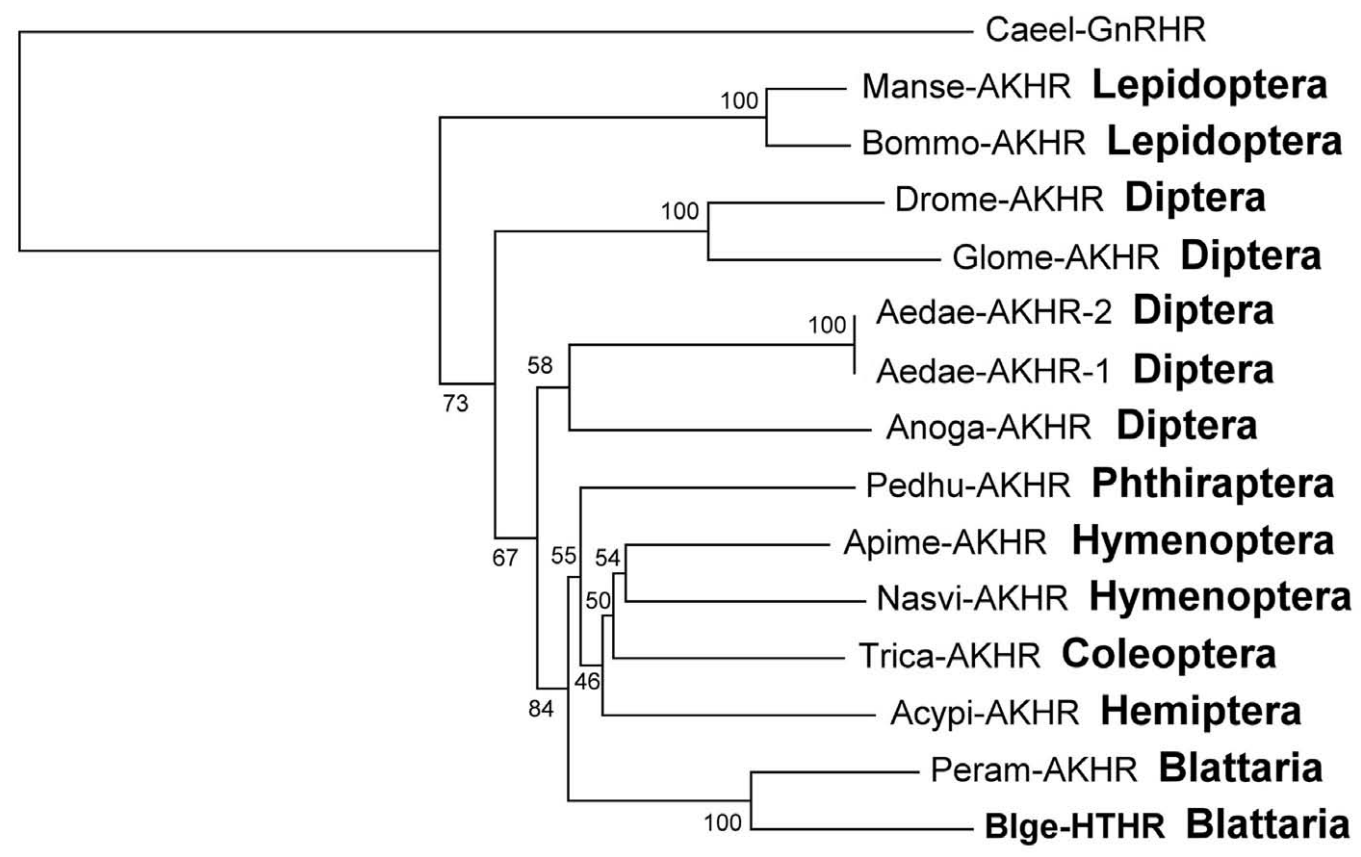

0.1

FIGURE 1 | (A) Alignment of the deduced amino acid sequences of the Blattella germanica HTH receptor (Blage-HTHR, GenBank GU591493) with the Periplaneta americana AKH receptor (Peram-AKHR, GenBank ABB20590). Identical amino acid residues are highlighted in gray. The seven transmembrane $\alpha$-helices are indicated by TM1-7. The dotted-line rectangle indicates an insertion present in both cockroach species. (B) Neighbor joining tree of $\mathrm{AKH}$ receptor insect sequences. The tree was generated using MEGA 5 with 1000 bootstrap replicates. The evolutionary distance is given in units of the number of amino acid substitutions per site. The abbreviated names of insect AKHR are: Acypi-AKHR (Acyrthosiphon pisum), Aedae-AKHR-1 and Aedae-AKHR2 (Aedes aegypti), Anoga-AKHR (Anopheles gambiae), Apime-AKHR (Apis mellifera), Blage-HTHR (Blattella germanica), Bommo-AKHR (Bombyx mori), Drome-AKHR (Drosophila melanogaster), Glomo-AKHR (Glossina morsitans), Manse-AKHR (Manduca sexta), Nasvi-AKHR (Nasonia vitripennis), Pedhu-AKHR (Pediculus humanus), Peram-AKHR (Periplaneta americana), and Trica-AKHR (Tribolium castaneum). Gonadotropin-releasing hormone receptor of Caenorhabditis elegans (Caeel-GnRHR) was used as outgroup. 
GAA-3' forward primer, and 5'-GGG AAA TGT CTT GTG AAC CAG GTC-3' reverse. The MEGAscript RNAi Kit (Ambion) was used to generate the dsRNA following the manufacturer's protocol. The dsRNA solution was stored at $-20^{\circ} \mathrm{C}$ until use. A dose of $1.5 \mu \mathrm{g}$ of the $528 \mathrm{bp}$ dsRNA targeting the Blage-HTHR mRNA was injected into the abdomen of newly emerged adults. As dsRNA control, the enhanced green fluorescence protein (EGFP) sequence was used at the same dose. Blage-HTHR expression in the fat body was determined by semiquantitative RT-PCR within the following days after dsRNA treatment.

To investigate the role of HTH against oxidative stress, we injected $1.5 \mu \mathrm{g}$ of both, dsHTHR and dsHTH, the latter being prepared according to the protocol previously described (Huang and Lee, 2011), into newly emerged male B. germanica. Blage-HTHR expression in the fat body and Blage-HTH expression in the whole head were determined by semiquantitative RT-PCR at the 10th day after dsRNA treatment.

\section{TREHALOSE DETERMINATION}

Trehalose in the hemolymph was determined following the protocol previously described (Huang and Lee, 2011). Briefly, $5 \mu 1$ hemolymph was obtained from the hind leg coxae. Then, the hemolymph was incubated overnight at $37^{\circ} \mathrm{C}$ with $2 \mu \mathrm{l}$ porcine kidney trehalase (Sigma) to convert trehalose into glucose. The amount of glucose in $50 \mu \mathrm{l}$ of the above solution was measured using the Glucose Assay Kit (Sigma). Glucose concentration was corrected by deducting the amount of glucose from the same supernatant prepared under identical conditions but without trehalase.

\section{OXIDATIVE STRESS TREATMENTS}

The insects were individually injected with $1 \mu$ l solution of $20 \mathrm{mM}$ PQ (paraquat: 1,1'-dimethyl-4,4'-bipyridylium dichloride hydrate) to cause oxidative stress, or with $1 \mu$ l Ringer saline as control. To study the effect of HTH against oxidative stress, the insects were injected with $1 \mu$ l solution containing synthetic HTH peptide (pGlu-Val-Asn-Phe-Ser-Pro-Gly-Trp-GlyThr- $\mathrm{NH}_{2}$ ) of B. germanica at various concentrations (10, 40, and $80 \mathrm{pmol}$ ) in combination with $20 \mathrm{nmol} \mathrm{PQ} \mathrm{(1:1} \mathrm{in} \mathrm{volume).} \mathrm{The}$ number of dead cockroaches were recorded every day. All chemicals used in the experiments and assays were obtained from Sigma-Aldrich Chemical Company (St. Louis, MO, USA) unless otherwise stated.

\section{LIPID PEROXIDATION MEASUREMENT}

We used lipid peroxidation as specific test to measure the level of oxidative stress. A total of $15 \mu \mathrm{l}$ hemolymph from a pool of eight specimens was collected at $4 \mathrm{~h}$ post inoculation of oxidative stress treatment and diluted with $30 \mu \mathrm{l}$ PBS containing 1\% butylated hydroxytoluene to prevent further oxidation of lipid during further processing. The diluted hemolymph was shaken vigorously and centrifuged $\left(10,000 \times g\right.$ for $10 \mathrm{~min}$ at $\left.4^{\circ} \mathrm{C}\right)$. To determine the levels of malondialdehyde as indicator of lipid peroxidation, the supernatants were processed using the OxiSelect TBARS (thiobarbituric acid reactive substances) assay kit (Cell Biolabs) following the manufacturer's instructions.

\section{STATISTICAL ANALYSIS}

The results in the figures represent the mean \pm SEM. Statistical analyses were performed using computing software R. Statistical significance of results were evaluated using unpaired $t$-test between two groups and one-way ANOVA followed by Tukey's HSD post hoc test for multiple comparisons. The analyses of survival curves were carried out using Kaplan-Meier test for median survival time, the Log-rank test for testing the differences among treatment groups, and the Kruskal-Wallis rank sum test for post-test calculations.

\section{RESULTS}

\section{CLONING AND EXPRESSION PATTERN OF BLAGE-HTH RECEPTOR}

Cloning of the HTH receptor (Blage-HTHR) cDNA of B. germanica was accomplished by a RT-PCR approach, combining the use of degenerate primers based on AKHR conserved motifs to obtain a partial sequence, and 5'-RACE and $3^{\prime}$-RACE experiments to complete it. These amplifications rendered a full-length cDNA of 1,769 bp encoding a predicted 406 amino acid protein (Blage-HTHR; GenBank accession number GU591493; Figure A1 in Appendix). According to BLAST searches, cDNA sequence of Blage-HTHR was very close (71.0\% identity, $81.5 \%$ similarity) to P. americana $\mathrm{AKH}$ receptor (Peram-AKHR; GenBank accession number ABB20590). In addition, the alignment of receptor proteins of the two cockroach species showed values of $62.4 \%$ identity and $72 \%$ similarity (Figure 1A). The deduced amino acid sequence of Blage-HTHR exhibits the typical structural features of $G$ protein-coupled receptors including an extracellular $\mathrm{N}$-terminus, seven transmembrane regions, and an intracellular C-terminus.

Neighbor-joining analysis of insect $\mathrm{AKH}$ receptor sequences gave a tree (Figure 1B) showing that Blage-HTHR clusters with Peram-AKHR from $P$. americana, as expected. Indeed, these two cockroach receptors have an additional consensus sequence in the intracellular C-terminus that is characteristic of them, and which is not present in other insect $\mathrm{AKH}$ receptor sequences (Figure A2 in Appendix). The sister group of the cockroach node is composed by phylogenetically distal hemimetabolous species (Phthiraptera, Hemiptera) and basal holometabolous species (Coleoptera, Hymenoptera), and these two nodes have the more modified holometabolous species (Lepidoptera and Diptera) as sister group. In general, the topology of the tree approximately follows the phylogenetic relationships of the insect orders currently established.

The expression of Blage-HTHR was detected and quantified in various tissues and stages (Figure 2). Concerning the adult, the expression was observed in practically all studied tissues, but the strongest expression was found in the fat body (Figure 2A). Conversely, expression in the ovary was practically absent, and that in the accessory glands was very faint. Comparing different life cycle stages (Figure 2B), expression was very low in embryos and relatively low in the first instar nymph. Values were much higher in the last instar (N6) nymph, and in the adult stage of both sexes.

\section{RNAI-MEDIATED KNOCKDOWN OF BLAGE-HTHR REDUCES THE TREHALOSEMIC EFFECT OF HTH}

We first studied the function of Blage-HTHR in B. germanica in relation to trehalose regulation by depleting its expression by 


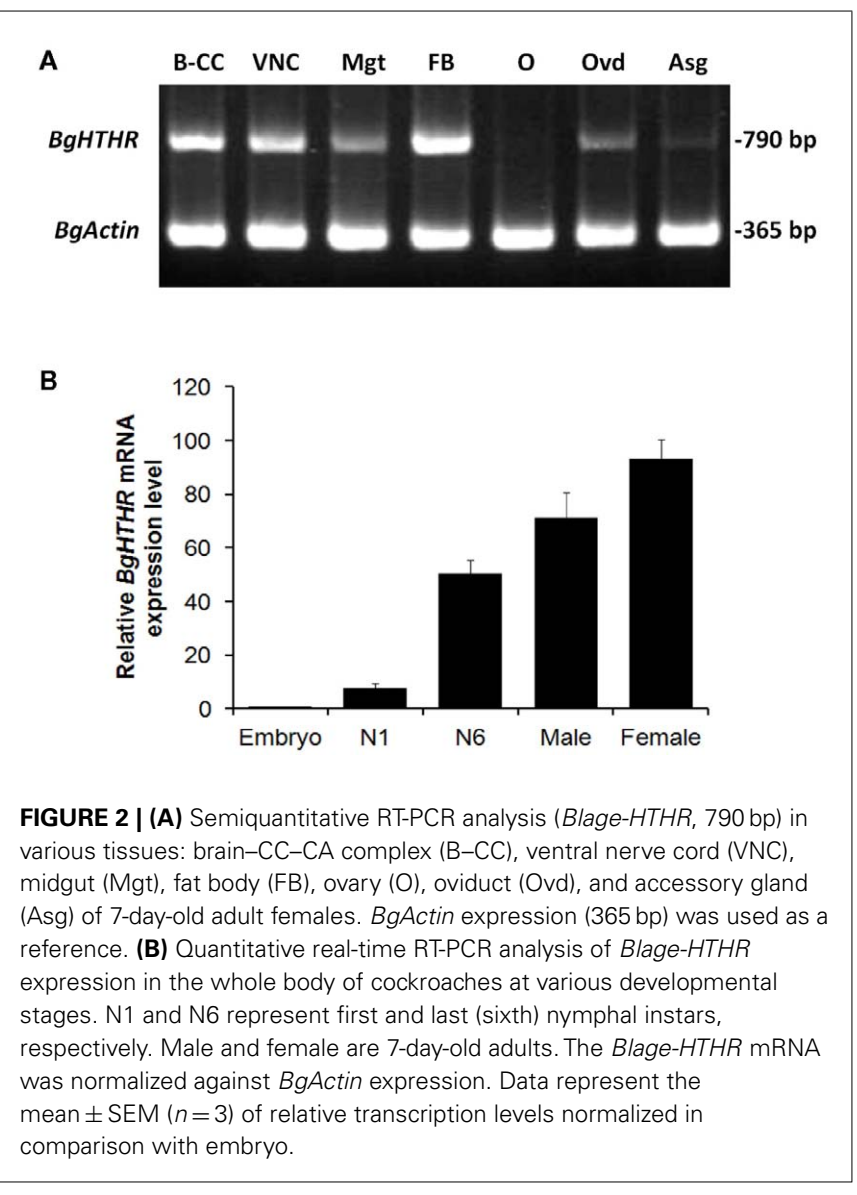

RNAi. Blage-HTHR mRNA was measured in the fat body 1, 5, and 10 days after the treatment with dsHTHR, and results (Figure 3A) showed that expression was clearly reduced in comparison with controls (dsEGFP-treated) on days 1 and 5, and it was practically undetectable on day 10, either in females as well as in males. The function of Blage-HTHR as HTH receptor was illustrated by the significant reduction of trehalose titers in the hemolymph after injection of HTH in dsHTHR-treated females (Figure 3B). The injection of $10 \mathrm{pmol}$ of $\mathrm{HTH}$ resulted in the increase of hemolymph trehalose up to $113 \% 2 \mathrm{~h}$ later in dsEGFP-treated females. In contrast, the hemolymph trehalose increased significantly less (49\%) in dsHTHR-treated females $2 \mathrm{~h}$ after the HTH injection. These results suggest that the remarkable increase of hemolymph trehalose elicited by HTH requires a high expression of its receptor, Blage-HTHR.

The titer of hemolymph trehalose displayed a steady increase and peaked at day 8 in the first reproductive cycle of female adults, either in controls (dsEGFP-treated) as in specimens treated with dsHTHR (Figure 3C), although the levels are lower in the latter. The hemolymph trehalose pattern is coincident with that of ovarian development. In adult males, the hemolymph trehalose levels practically do not fluctuate, either in controls (dsEGFPtreated) as in specimens treated with dsHTHR (Figure 3D); again, the levels were significantly lower in dsHTHR-treated specimens (Figure 3D).

\section{ROLE OF HTH AS RELIEVER OF OXIDATIVE STRESS}

Subsequently, we studied the potential roles of $\mathrm{HTH}$ as reliever of oxidative stress. Given that female B. germanica exhibits a varied ability against oxidative stress during the reproductive cycle

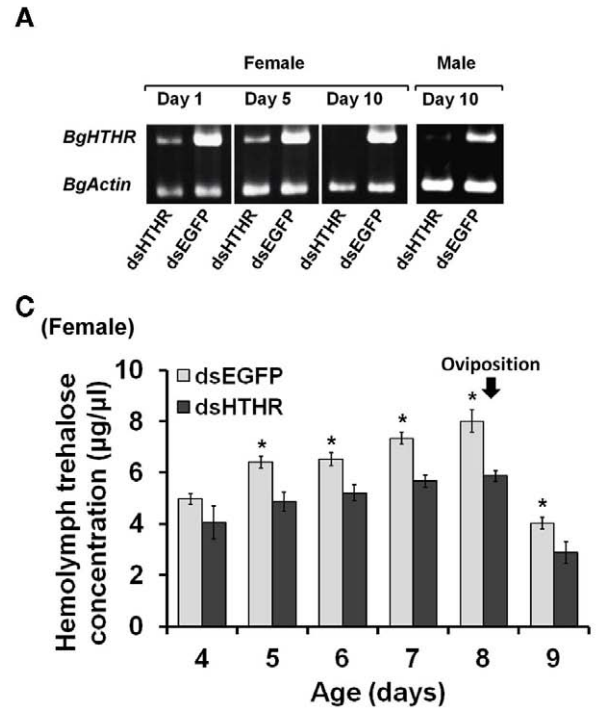

FIGURE 3 | Effects of silencing Blage-HTHR on hemolymph trehalose levels in Blattella germanica. dsHTHR or dsEGFP were injected in newly emerged adults. (A) Semiquantitative RT-PCR analysis showing the down-regulation of Blage-HTHR expression in the fat body 1 day after injection of the corresponding dsRNA. (B) Silencing effect of Blage-HTHR on hemolymph trehalose levels after treatment with $\mathrm{HTH}(10 \mathrm{pmol} ; n=16) 0$ and
B

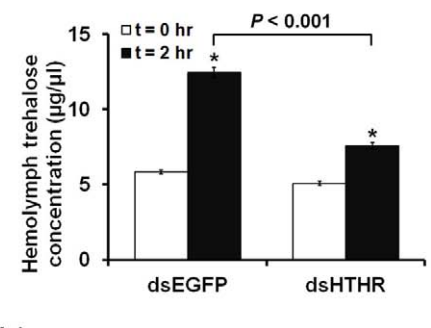

(Male)

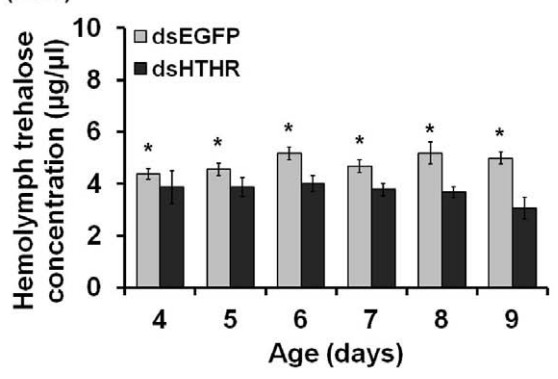

$2 \mathrm{~h}$ after $\mathrm{HTH}$ treatment. ${ }^{*}$ Indicates significant differences between 0 and $2 \mathrm{~h}$ after HTH treatment $(P<0.05$, two-tailed Student's $t$-test). (C) Pattern of hemolymph trehalose in dsHTHR-treated adult females during the first reproductive cycle ( $n=8$, except at the age of 4 days, where $n=4)$. (D) Pattern of hemolymph trehalose in dsHTHR-treated adult males $(n=6)$. ${ }^{*} P<0.05$ is using two-tailed Student's $t$-test. 
(unpublished data), we chose only males to conduct the following experiments. The injection of $20 \mathrm{nmol}$ of PQ on 10-day-old male adults caused a mortality that rapidly increased with time, thus median survival time was attained on day 4 (Figure 4A). However, exogenous HTH at 10, 40, and 80 pmol concentrations significantly reduced the speed of mortality increase, with median survival time attained around day 7 (Figure 4A), thus, the protective effect of HTH did not show significant differences among the three concentrations used.

To further assess the possible protective role of $\mathrm{HTH}$ against oxidative stress, the expression of Blage-HTHR after PQ treatment was studied (Figure 4B). Injection of PQ (20 nmol) caused a significant reduction of the Blage-HTHR expression in the fat body $4 \mathrm{~h}$ later. Mean value of Blage-HTHR mRNA was also decreased in the specimens injected with PQ and 40 pmol HTH, which extended the life span under the provoked oxidative stress. But the level of Blage-HTHR in PQ co-injected with HTH was not significantly different from that in either control or PQ treatment.

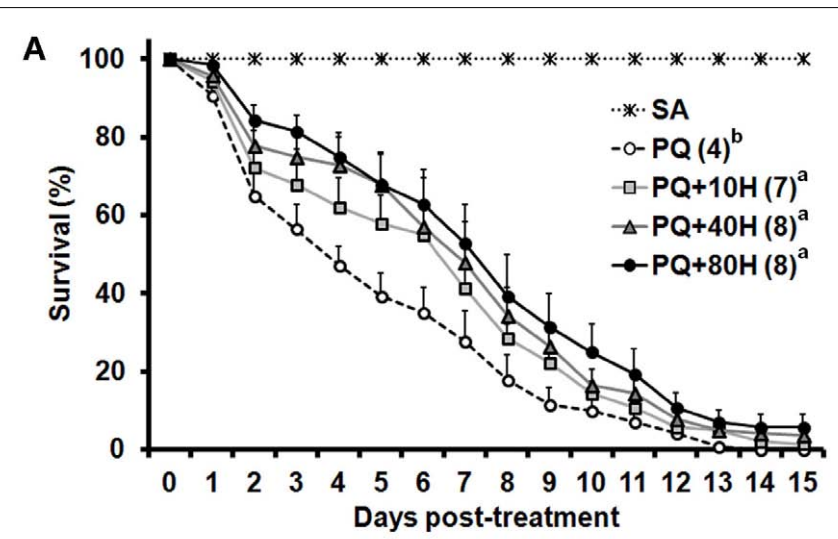

B

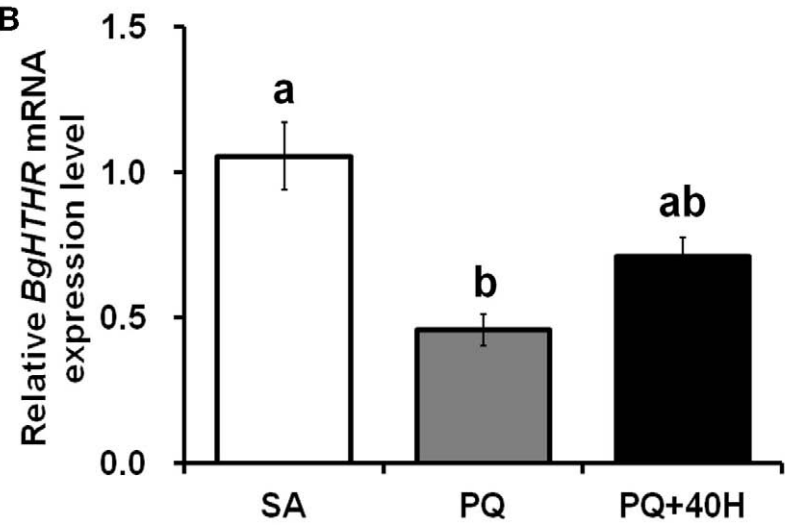

FIGURE 4 | (A) Survival curve of male adults of Blattella germanica after treatment with paraquat $(\mathrm{PQ})$ and $\mathrm{HTH}(\mathrm{H})$. SA, injection with saline (control); $\mathrm{PQ}$, injection with $1 \mu \mathrm{l}$ of $20 \mathrm{mM} \mathrm{PO} ; 10 \mathrm{H}, 40 \mathrm{H}$, and $80 \mathrm{H}$, injection with various concentrations (pmol) of $\mathrm{HTH}$. The median survival time (days) is indicated in the parenthesis of each treatment group. (B) Quantitative real-time PCR analyses on mRNA expression of Blage-HTHR in fat body of adult male of $B$. germanica $4 \mathrm{~h}$ after the treatment. Expression levels were calculated after normalizing against BgActin expression in each sample. Results are indicated as the mean \pm SEM ( $n=3$ per treatment group).

\section{RNAi-SILENCED HTH SIGNALING REDUCES ANTI-OXIDATIVE PROTECTION}

We further assessed the protective role of $\mathrm{HTH}$ on oxidative stress in specimens were Blage-HTH and Blage-HTHR mRNA levels had been simultaneously depleted by RNAi. A treatment of $1.5 \mu \mathrm{g}$ of dsHTH and $1.5 \mu \mathrm{g}$ of dsHTHR (dsHTH/R) was administered to newly emerged males of $B$. germanica, and the corresponding expression of Blage-HTH and Blage-HTHR was undetectable 10 days later in the tissues were these transcripts are most expressed: Blage-HTH in the head and Blage-HTHR in the fat body (Figure 5A). There were no lethal effects when the expression of both genes was knocked down, as all specimens survived in apparently healthy conditions at least during 25 days after the dsRNA injections. However, PQ treatment elicited a faster mortality in the specimens knockdown for Blage-HTH and Blage-HTHR (median survival time was attained on day 2 after PQ treatment), than in controls (dsEGFP-treated; median survival time attained on day 5; Figure 5B). Moreover, a dose of 40 pmol of HTH significantly relieved the detrimental effect elicited by PQ in control specimens (median survival time attained on day 8), but this "rescue" effect of HTH was milder in the specimens knockdown for Blage-HTH and Blage-HTHR (median survival time attained on day 3; Figure 5B).

Finally, the anti-oxidative stress function of Blage-HTH and Blage-HTHR was further demonstrated by measuring malondialdehyde as indicator of lipid peroxidation under oxidative stress caused by PQ, using specimens where Blage-HTH and BlageHTHR expression had been depleted by RNAi, and applying exogenous HTH. Results (Figure 5C) show that without PQ, the malondialdehyde titer in the group of specimens knockdown for Blage-HTH and Blage-HTHR was higher than that of the dsEGFP-treated group. PQ elicited and increased the titer of malondialdehyde in both groups, but differences between them were not statistically significant. Finally, exogenous HTH significantly counteracted the effects of PQ in the dsEGFP-treated group, but not in the group of Blage-HTH and Blage-HTHR knockdowns.

\section{DISCUSSION}

About 50 peptides belonging to the AKH family have been identified in insects (Gäde, 2009) since the first hyperglycemic factor was discovered in the cockroach $P$. americana (Steele, 1961). The physiological function of these peptides on energy homeostasis as well as on signal transduction at the cellular level have been thoroughly reviewed by a number of authors (Van der Horst et al., 2001; Gäde and Auerswald, 2003; Schooley et al., 2005). However, studies at molecular level of AKH receptors have been conducted more recently in the fruit fly $D$. melanogaster and in the silkmoth B. mori (Staubli et al., 2002). In cockroaches, only the AKH receptor cDNA of $P$. americana has been characterized (Hansen et al., 2006; Wicher et al., 2006). The present contribution reports the characterization of the HTH receptor in another cockroach, $B$. germanica, which is structurally close to that of P. americana $\mathrm{AKH}$ receptor (Figure 1).

Alignment of all known AKH/HTH receptor protein sequences shows a large region of evolutionary conserved residues in the transmembrane domain, but a highly diversified region in the Cterminus, which is associated to intracellular signaling (Figure A2 


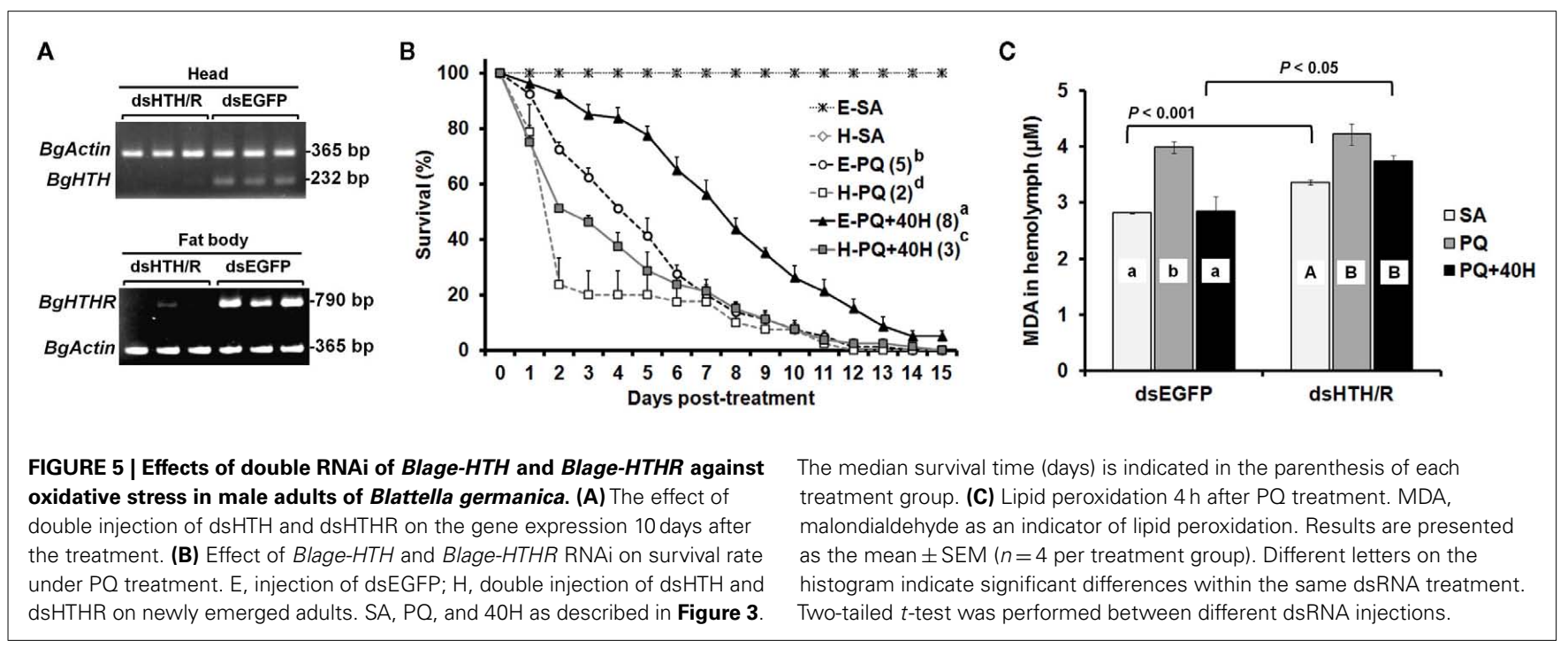

in Appendix). The phylogenetic analysis indicates that the intracellular C-terminus has considerably diverged in different insect orders, and this might have derived in different signal transduction mechanisms in different orders. Interestingly, an additional sequence at the beginning of the intracellular C-terminus in cockroaches might be related to the export of the receptor to the cell membrane, as an equivalent sequence in the C-terminal end of the AKH receptor of $B$. mori serves for this function (Huang et al., 2011).

The most abundant expression of Blage-HTHR in the fat body among the tissues tested (Figure 2A) is in agreement with the fact that HTH is the major regulator of energy metabolism in insects. This is also supported by the increasing expression level of Blage-HTHR from nymphal to adult stage which coincides with increasing locomotion which occurs in parallel (Yang et al., 2009), and which requires increasing energy expenditure. Our previous results showed that depletion of Blage-HTH expression results in a delay of oviposition in virgin females of B. germanica (Huang and Lee, 2011). In the present study, we observed Blage-HTHR expression in the oviduct, but not in ovary (Figure 2A), which suggests that Blage-HTH release could stimulate the contraction of oviduct muscles during basal oocyte extrusion. Indeed, a myoactive effect of AKHs on the dorsal vessel has been demonstrated in P. americana (Scarborough et al., 1984).

The canonical function of Blage-HTHR as $\mathrm{HTH}$ receptor in $B$. germanica has been demonstrated by injecting $\mathrm{HTH}$ in specimens where Blage-HTHR had been depleted by RNAi, and observing that mobilization of trehalose in the hemolymph was impaired (Figure 3B). Equivalently, the malaria mosquito A. gambiae with lowered expression of $\mathrm{AKH}$ receptor (Anoga-AKHR) also fails to mobilize the glycogen reserves in the fat body after injection of Anoga-AKH-I (Kaufmann and Brown, 2008). However, the occurrence of a significant elevation of hemolymph trehalose in dsHTHR-treated group was likely considered as incomplete silencing of Blage-HTHR expression because RNAi does not completely knockout gene expression. In addition, studies in vitro demonstrate that reduced expression of the Bombyx AKHR on the cell membrane results in lower levels of intracellular cAMP (cyclic adenosine monophosphate) formation after AKH stimulation (Huang et al., 2011). Therefore, a maximal action of $\mathrm{HTH}$ would require an adequate expression of its receptor to trigger signal transduction.

RNA interference-mediated depletion of Blage-HTHR mRNA was found to decrease hemolymph trehalose levels in the adult of both sexes (Figures 3C,D), although the female displays fluctuations of trehalose related to the reproductive cycle. Our present results of Blage-HTHR RNAi (Figure 3C) supports our previous hypothesis postulating that HTH induces hemolymph trehalose mobilization during the reproductive cycle in virgin females (Huang and Lee, 2011). The results suggest that HTH plays a critical role in carbohydrate homeostasis, as occurs in $\mathrm{AKH}$ cell-deficient $D$. melanogaster that has relatively low hemolymph trehalose levels (Lee and Park, 2004).

The involvement of $\mathrm{AKH}$ peptides in antioxidant protection in insects was inferred from the increase in $\mathrm{AKH}$ titer after exposure to oxidative stress (Kodrik et al., 2007; Vecera et al., 2007). The same authors showed that exogenous AKH application enhanced the efflux of reduced glutathione to the hemolymph and maintained the normal levels of protein carbonyl, a marker of oxidative stress. In the present study, we have fully demonstrated the protective role of HTH upon oxidative stress under different experimental approaches, first, through experiments of co-injection of PQ and HTH on control specimens (Figure 4A), and secondly, in specimens with the expression levels of BlageHTH and Blage-HTHR depleted by RNAi (Figure 5B). These results provide a new and relevant information supporting that Blage-HTH potentiates a stress hormone on anti-oxidative stress response in B. germanica. The promoter of oxidative stress used in our experiments has been PQ, which acts on the redox-cycling reactions, thus eliciting free radicals as well as causes oxidative modification of macromolecules, such as lipid peroxidation (Hassan, 1984). Accordingly, we examined lipid peroxidation in the hemolymph $4 \mathrm{~h}$ after PQ treatment (Figure 5C). Levels of lipid 
peroxidation in the hemolymph increased significantly after PQ injection in dsEGFP and $\mathrm{dsHTH} / \mathrm{R}$ groups. However, the coinjection of HTH maintained the lipid peroxidation at a normal level in controls but not in the Blage-HTH and Blage-HTHR knockdowns, which suggests that HTH lowers the oxidative damage in B. germanica. A likely mechanism might be that HTH induces the efflux of glutathione into the hemolymph, as demonstrated in other insect AKHs (Kodrik et al., 2007; Vecera et al., 2007). However, the significant reduction of Blage-HTHR expression after PQ treatment (Figure 4B), suggests that the oxidative stress directly impairs its expression. The less severe reduction of Blage-HTHR expression observed after co-injecting PQ and HTH (Figure 4B) might be the outcome of downstream response induced by $\mathrm{HTH}$ to relieve the oxidative damage. Moreover, genome-wide analysis in D. melanogaster after PQ treatment have shown that cytochrome P450 genes become up- and

\section{REFERENCES}

Belles, X. (2010). Beyond Drosophila: RNAi in vivo and functional genomics in insects. Annu. Rev. Entomol. 55, 111-128.

Candy, D. J. (2002). Adipokinetic hormones concentrations in the haemolymph of Schistocerca gregaria, measured by radioimmunoassay. Insect Biochem. Mol. Biol. 32, 1361-1367.

Chaudhuri, A., Bowling, K., Funderburk, C., Lawal, H., Inamdar, A., Wang, Z., and O'Donnell, J. M. (2007). Interaction of genetic and environmental factors in a Drosophila parkinsonism model. J. Neurosci. 27, 2457-2467.

Gäde, G. (2009). Peptides of the adipokinetic hormone/red pigmentconcentrating hormone family: a new take on biodiversity. Ann. N. Y. Acad. Sci. 1163, 125-136.

Gäde, G., and Auerswald, L. (2003). Mode of action of neuropeptides from the adipokinetic hormone family. Gen. Comp. Endocrinol. 132, 10-20.

Girardot, F., Monnier, V., and Tricoire, H. (2004). Genome wide analysis of common and specific stress responses in adult Drosophila melanogaster. BMC Genomics 5, 74. doi:10.1186/1471-2164-5-74

Granett, J., and Leeling, N. C. (1972). A hyperglycemic agent in the serum of DDT-prostrate American cockroaches, Periplaneta americana. Ann. Entomol. Soc. Am. 65, 299-302.

Halliwell, B., and Gutteridge, J. M. C. (2007). Free Radicals in Biology and Medicine, 4th Edn. Oxford: Clarendon Press.

Hansen, K. K., Hauser, F., Cazzamali, G., Williamson, M., and Grimmelikhuijzen, C. J. P. (2006). Cloning and characterization of the adipokinetic hormone receptor from the cockroach Periplaneta americana. Biochem. Biophys. Res. Commun. 343, 638-643.

Hassan, H. M. (1984). Exacerbation of superoxide radical formation by paraquat. Meth. Enzymol. 105, 523-532.

Huang, H., Deng, X., He, X., Yang, W., Li, G., Shi, Y., Shi, L., Mei, L., Gao, J., and Zhou, N. (2011). Identification of distinct c-terminal domains of the Bombyx adipokinetic hormone receptor that are essential for receptor export, phosphorylation and internalization. Cell. Signal. 23, 1455-1465.

Huang, J.-H., and Lee, H.-J. (2011). RNA interference unveils functions of the hypertrehalosemic hormone on cyclic fluctuation of hemolymph trehalose and oviposition in the virgin female Blattella germanica. J. Insect Physiol. 57, 858-864.

Kaufmann, C., and Brown, M. R. (2008). Regulation of carbohydrate metabolism and flight performance by a hypertrehalosaemic hormone in the mosquito Anopheles gambiae. J. Insect Physiol. 54, 367-377.

Kodrik, D. (2008). Adipokinetic hormone functions that are not associated with insect flight. Physiol. Entomol. 33, 171-180.

Kodrik, D., Krishnan, N., and Habustova, O. (2007). Is the titer of adipokinetic peptides in Leptinotarsa decemlineata fed on genetically modified potatoes increased by oxidative stress? Peptides 28, 974-980.

Kodrik, D., and Socha, R. (2005). The hormone titre in the insect body. Pest Manag. Sci. 61, 1077-1082.

Lee, G., and Park, J. H. (2004). Hemolymph sugar homeostasis and starvation-induced hyperactivity affected by genetic manipulations of effect of insecticide on adipokinetic

down-regulated (Girardot et al., 2004), and it has been additionally reported that HTH stimulates cytochrome P450 expression in the cockroach Blaberus discoidalis (Lu et al., 1995). Therefore, we further speculate that HTH might induce the expression of P450 genes, thus contributing to decrease the oxidative stress in $B$. germanica.

\section{ACKNOWLEDGMENTS}

We are grateful to Chin-Cheng Yang for his help with the phylogenetic analyses. Support for this research was provided by National Science Council of Taiwan (NSC 97-2313-B-002-031 and NSC 100-2923-B-002-002 to How-Jing Lee; NSC 98-2917-I-002-147 to Jia-Hsin Huang for working one year in Barcelona), and the Spanish MICINN (grant CGL2008-03517/BOS to Xavier Bellés) and by the CSIC (grant 2010TW0019, from the Formosa program, to Xavier Bellés).

the adipokinetic hormone-encoding gene in Drosophila melanogaster. Genetics 167, 311-323.

Lee, H. J., and Wu, Y. L. (1994). Mating effects on the feeding and locomotion of the German cockroach, Blattella germanica. Physiol. Entomol. 19, 39-45.

Lu, K.-H., Bradfield, J. Y., and Keeley, L. L. (1995). Hypertrehalosemic hormone-regulated gene expression for cytochrome $\mathrm{P} 4504 \mathrm{Cl}$ in the fat body of the cockroach Blaberus discoidalis. Arch. Insect Biochem. Physiol. 28, 79-90.

Nicholas, K. B., Nicholas, H. B., and Deerfield, D. W. (1997). GeneDoc: analysis and visualization of genetic variation. Embnet News 4, 14.

Pfaffl, M. W. (2001). A new mathematical model for relative quantification in real-time RT-PCR. Nucleic Acids Res. 29, e45.

Samaranayaka, M. (1974). Insecticideinduced release of hyperglycaemic and adipokinetic hormones of Schistocerca gregaria. Gen. Comp. Endocrinol. 24, 424-436.

Scarborough, R. M., Jamieson, G. C., Kalish, F., Kramer, S. J., McEnroe, G. A., Miller, C. A., and Schooley, D. A. (1984). Isolation and primary structure of two peptides with cardioacceleratory and hyperglycemic activity from the corpora cardiaca of Periplaneta americana. Proc. Natl. Acad. Sci. U.S.A. 81, 5575-5579.

Schooley, D. A., Horodyski, F. M., and Coast, G. M. (2005). "Hormones controlling homeostasis in insects," in Comprehensive Molecular Insect Science, eds L. I. Gilbert, K. Iatrou, and S. S. Gill (Amsterdam: Elsevier Press), 493-550.

Staubli, F., Jorgensen, T. J. D., Cazzamali, G., Williamson, M., Lenz, C., Sondergaard, L., Roepstorff, P., and Grimmelikhuijzen, C. J. P.
(2002). Molecular identification of the insect adipokinetic hormone receptors. Proc. Natl. Acad. Sci. U.S.A. 99, 3446-3451.

Steele, J. E. (1961). Occurrence of a hyperglycaemic factor in the corpus cardiacum of an insect. Nature 192, 680-681.

Tamura, K., Peterson, D., Peterson, N., Stecher, G., Nei, M., and Kumar, S. (2011). MEGA5: molecular evolutionary genetics analysis using maximum likelihood, evolutionary distance, and maximum parsimony methods. Mol. Biol. Evol. 28, 2731-2739.

Thompson, J. D., Gibson, T. J., Plewniak, F., Jeanmougin, F., and Higgins, D. G. (1997). The CLUSTAL_X windows interface: flexible strategies for multiple sequence alignment aided by quality analysis tools. Nucleic Acids Res. 25, 4876-4882.

Valko, M., Leibfritz, D., Moncol, J., Cronin, M. T., Mazur, M., and Telser, J. (2007). Free radicals and antioxidants in normal physiological functions and human disease. Int. J. Biochem. Cell Biol. 39, 44-84.

Van der Horst, D. J., Van Marrewijk, W. J. A., and Diederen, J. H. B. (2001). "Adipokinetic hormones of insect: release, signal transduction, and responses," in International Review of Cytology - a Survey of Cell Biology, ed. K. W. Jeon (San Diego, CA: Academic Press), 179-240.

Vecera, J., Krishnan, N., Alquicer, G., Kodrik, D., and Socha, R. (2007) Adipokinetic hormone-induced enhancement of antioxidant capacity of Pyrrhocoris apterus hemolymph in response to oxidative stress. Comp. Biochem. Physiol. C Toxicol. Pharmacol. 146, 336-342. 
Velki, M., Kodrik, D., Vecera, J., Hackenberger, B. K., and Socha, R. (2011). Oxidative stress elicited by insecticides: a role for the adipokinetic hormone. Gen. Comp. Endocrinol. 172, 77-84.

Wicher, D., Agricola, H. J., Sohler, S., Gundel, M., Heinemann, S. H., Wollweber, L., Stengl, M., and Derst, C. (2006). Differential receptor activation by cockroach adipokinetic hormones produces differential effects on ion currents, neuronal activity, and locomotion. J. Neurophysiol. 95, 2314-2325.

Yang, Y. Y., Wen, C.-J., Mishra, A., Tsai, C.-W., and Lee, H.-J. (2009). Development of the circadian clock in the German cockroach, Blattella germanica. J. Insect Physiol. 55, 469-478.

Conflict of Interest Statement: The authors declare that the research was conducted in the absence of any commercial or financial relationships that could be construed as a potential conflict of interest.

Received: 28 October 2011; paper pending published: 18 November 2011; accepted: 20 December 2011; published online: 09 January 2012.

Citation: Huang J-H, Bellés $X$ and Lee $H-J$ (2012) Functional characterization of hypertrehalosemic hormone receptor in relation to hemolymph trehalose and to oxidative stress in the cockroach Blattella germanica. Front. Endocrin. 2:114. doi: 10.3389/fendo.2011.00114

This article was submitted to Frontiers in Experimental Endocrinology, a specialty of Frontiers in Endocrinology.

Copyright (ㄷ 2012 Huang, Bellés and Lee. This is an open-access article distributed under the terms of the Creative Commons Attribution Non Commercial License, which permits non-commercial use, distribution, and reproduction in other forums, provided the original authors and source are credited. 
31 CTTATGGCTGGAGGAGGCGGAAAAATCTGCAGTGTTAGAAAAAGCGATAACATTCTAAGAACAAACTGAATGATTAGACAAATCAAATGT

121 TCGAGAATTTATTCAACGAAGATTGAAGTGATGAACGAATTAGTTCTAACAGTGAAATGGAAGAGACGAAGTGATGATTCGCTTAGTGAA

211 ACAGTGGCATGGAAGAATTTGGGTGTTGGTAATCTTGATTCATCTTTCAACTGTCAACATTTTGACATGAGCATTTGACAGGAACCCAAG

301 ATGACGACTACAGAGCTGCCCCGTGAGCAGCAGCTGACTGAGGACATGACTTTTGGCTCCATACATAAGTTATGTATCGCCACGTATTGC

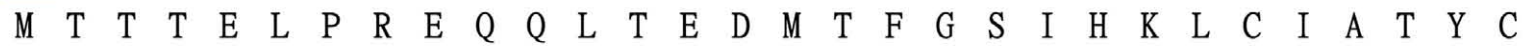

391 GTTCTCATGACTGTATCTGCAATTGGCAACATCACGGTTCTGGTCAACATACTCAAGAGACGCCGCAACCTTCGTTTTGGGAACAACTAC

$\begin{array}{llllllllllllllllllllllllllllll}\mathrm{V} & \mathrm{L} & \mathrm{M} & \mathrm{T} & \mathrm{V} & \mathrm{S} & \mathrm{A} & \mathrm{I} & \mathrm{G} & \mathrm{N} & \mathrm{I} & \mathrm{T} & \mathrm{V} & \mathrm{L} & \mathrm{V} & \mathrm{N} & \mathrm{I} & \mathrm{L} & \mathrm{K} & \mathrm{R} & \mathrm{R} & \mathrm{R} & \mathrm{N} & \mathrm{L} & \mathrm{R} & \mathrm{F} & \mathrm{G} & \mathrm{N} & \mathrm{N} & \mathrm{Y}\end{array}$

481 ATGTTCATGCATCTTGCCATAGCAGACCTATTGGTGACTTTTCTCATGATGCCGCTAGAAATCGGCTGGAATGCAACAGTGTCTTGGAGA

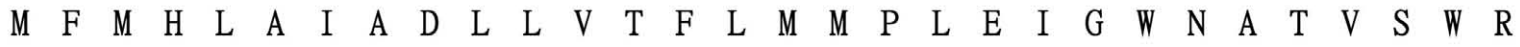

571 GCCGGTGACGCTGCTTGCAGAGTGATGTCGTTCTTCAGGATATTCGGCCTCTATCTGTCTAGCTTCGTAATTGTGTGCATCAGTCTGGAC

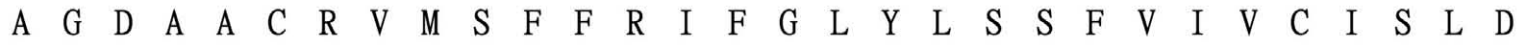

661 CGCTGTTTCGCCATTCTAAGGCCCATGTCGAACGTCGTCAACGTCGCCAAACGCAGCAGAGTAATGCTGACCACTGCCTGGTCATTGGCC

$\begin{array}{llllllllllllllllllllllllllllll}\mathrm{R} & \mathrm{C} & \mathrm{F} & \mathrm{A} & \mathrm{I} & \mathrm{L} & \mathrm{R} & \mathrm{P} & \mathrm{M} & \mathrm{S} & \mathrm{N} & \mathrm{V} & \mathrm{V} & \mathrm{N} & \mathrm{V} & \mathrm{A} & \mathrm{K} & \mathrm{R} & \mathrm{S} & \mathrm{R} & \mathrm{V} & \mathrm{M} & \mathrm{L} & \mathrm{T} & \mathrm{T} & \mathrm{A} & \mathrm{W} & \mathrm{S} & \mathrm{L} & \mathrm{A}\end{array}$

751 ACTGTTTGTAGTTTACCTCAGGTGTTCATCTTCCACGTACAACAGCATCCCGTTTTCACATGGTATGAGCAGTGTTTGGACTTCGACATG

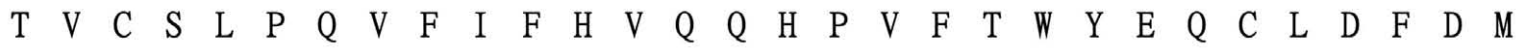

841 TTTCOGACTCAGCTGTACCAGTTCTGGTACAGGATATTAAATATGGTTCTAGTGTACGGTTTCCCACTCCTCGTCATTTTCATCTCATAC

$\begin{array}{llllllllllllllllllllllllllllll}\text { F } & P & T & Q & L & Y & Q & F & W & Y & R & I & L & N & M & V & L & V & Y & G & F & P & L & L & V & I & F & I & S & Y\end{array}$

931 GCCTGTATCCTCACAGAGATTTTTCGCAGATATCAGCTCAGTTCAGACGAAAACTTCCGGAGATCGAGCCTTGTGTTCCTGAACAGAGCC

$\begin{array}{llllllllllllllllllllllllllllll}A & C & I & L & T & E & I & F & R & R & Y & Q & L & S & S & D & E & N & F & R & R & S & S & L & V & F & L & N & R & A\end{array}$

1021 AAAAATAGGACGCTCAAAATGGCCATCATAATATTCGTAGTGTTCTTCATCTGCTGGACTCCCTACTATGTGATGTGTCTCTGGTACTGG

$\begin{array}{lllllllllllllllllllllllllllllll}\mathrm{K} & \mathrm{N} & \mathrm{R} & \mathrm{T} & \mathrm{L} & \mathrm{K} & \mathrm{M} & \mathrm{A} & \mathrm{I} & \mathrm{I} & \mathrm{I} & \mathrm{F} & \mathrm{V} & \mathrm{V} & \mathrm{F} & \mathrm{F} & \mathrm{I} & \mathrm{C} & \mathrm{W} & \mathrm{T} & \mathrm{P} & \mathrm{Y} & \mathrm{Y} & \mathrm{V} & \mathrm{M} & \mathrm{C} & \mathrm{L} & \mathrm{W} & \mathrm{Y} & \mathrm{W}\end{array}$

1111 ATAGACCAGCAGTCTGCTGAAAAGTTGACTTGCGCGTGAGAAAAGGCCTGTTCCTGTTTGCTTGTACCAATTCCTGTATGAACCCGATT

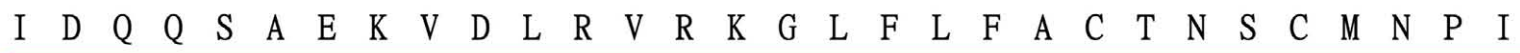

1201 GTGTACGGGTACTTCAACTTCCGTTCAGGACGGGGAAGTGGTTATGGTGCAACAAGACCAGGGCAGCAGTTACAGCATCATCAAATAACT

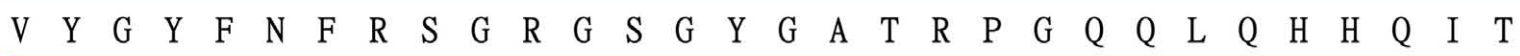

1291 GCATTGAGCAATAACTCAACGGGAGTTAACAGCCGAAGGGGAAGCAACTGCAGCAGCATCTATCGGGACAACAGCAACCAGAGCATGTCC

$\begin{array}{lllllllllllllllllllllllllllllll}A & L & S & N & N & S & T & G & V & N & S & R & R & G & S & N & C & S & S & I & Y & R & D & N & S & N & Q & S & M & S\end{array}$

1381 TGGAATCGCCGAAGCAGCCATGAAACAGAAATGCACGCCAATAACAATCGAGACGAAAATCACTTACACCCCAACTCAGCTGCGAACCAC

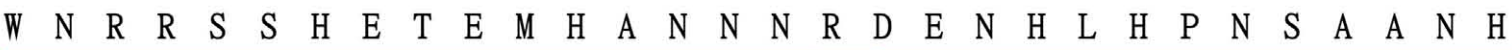

1471 AATTTGCGAACTACAGTATCTACTGTGAGTGAAGTTCCTGAAGCAAGATGAGGTTTACTCCTTGTGCTTCGAAATTATACTATCAGTGAT

$\begin{array}{llllllllllllllllllllllllllll}N & L & R & T & T & V & S & T & V & S & E & V & P & E & A & R & *\end{array}$

1561 AAACATTGCCAATGTAATTAAACATTAATGTTCTAGTCTAAGACTTGACATGACCTGGTTCACAAGACATTTCCCAAGAGAGTGAACAAA 1651 ACAATTGAATTGGTATAAACATGTGTTAAACTAATGTACAATACACTCACTCAACAGTTTTACACAAAAATAATAATTTATTATTTTTTG 1741 CCACTAAAAAAAAAAAAAAAAAAAAAAAA-3’

FIGURE A1 | Nucleotide sequence of cDNA encoding the open-reading frame for $B$. germanica HTHR with flanking $5^{\prime}$ - and 3'-UTR sequences (GenBank accession number GU591493). Start and stop codon are indicated by yellow background. The primer pair for
qRT-PCR is shown in underlined bold type. The primer pair for semiquantitative PCR is boxed. The fragment encompassed by the dsHTHR used in the RNAi experiments is shown in green background. 


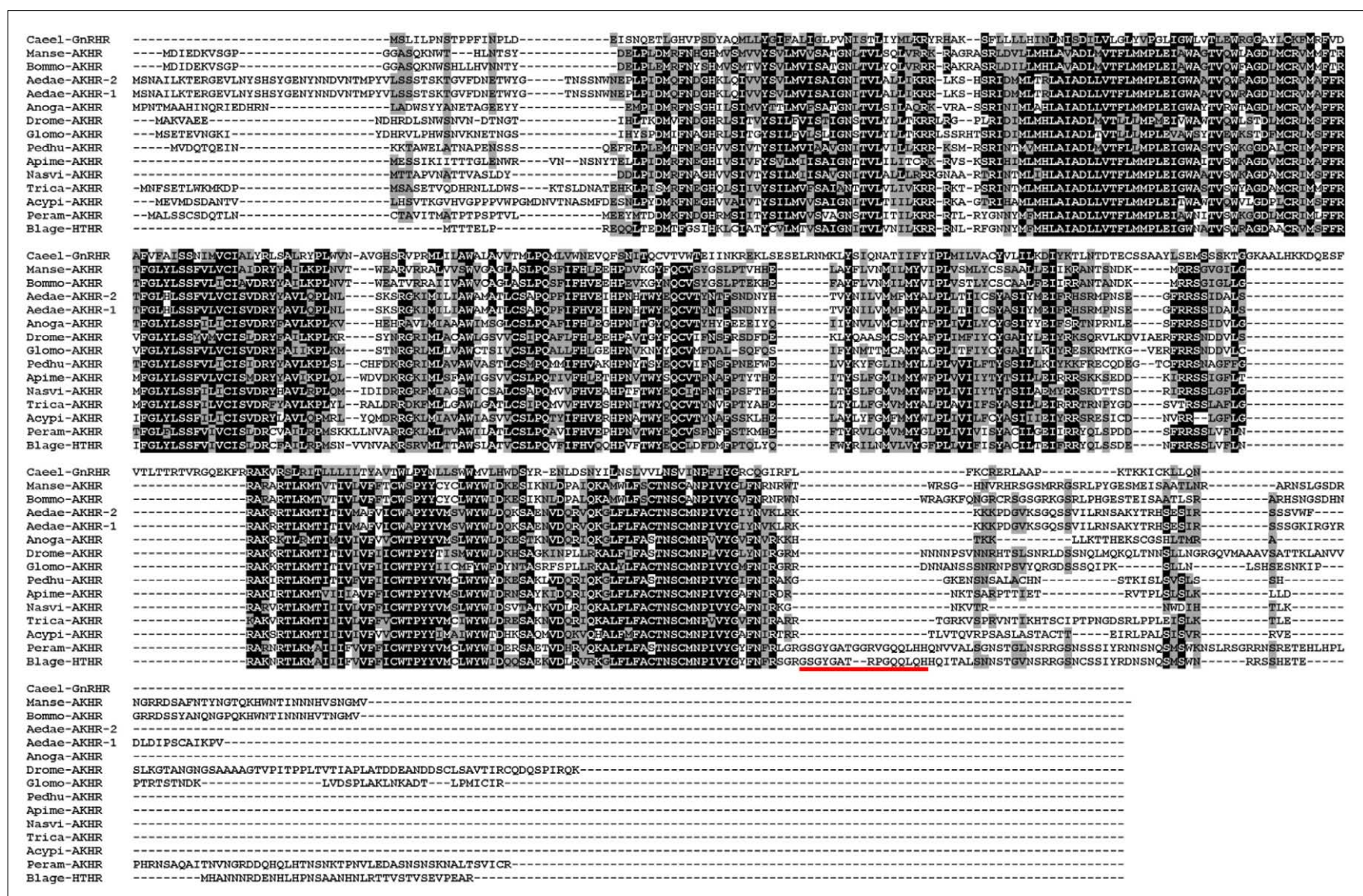

FIGURE A2 | Alignment of the AKH receptor protein sequences from insects and gonadotropin-releasing hormone receptor of Caenorhabditis elegans used in the phylogenetic analysis. The

alignment was carried out using ClustalW program. A black background indicates the amino acids are identical among species, whereas a gray background shows similarity among residues. The red underline indicates the insertion present in both cockroach species. The abbreviated names of sequences used are provided in Figure 1B. 